http://dx.doi.org/10.12795/PH.1990.v05.i01.18

\title{
Dificultades de aplicación de las primeras investigaciones de la «estética de la recepción» a los cuentos de Grimm ${ }^{1}$
}

Fernando Magallanes

La recepción de la literatura, como estudio de la supervivencia de la obra literaria, se plantea la pregunta de cómo y por qué sobrevive una obra de arte a la situación, circunstancias y relaciones en las cuales se produjo. Y la respuesta que se ofrece es que la obra permanece en tanto es actual; no por ser documento del pasado, sino por poder ser aplicada al presente.

En su método de estudio, la actual «Estética de la Recepción» se ocupa de los problemas inherentes a la recepción de la obra literaria a través del lector. No se trata, por tanto, de indagar acerca del contenido social de la obra literaria o de su estructura estética, sino de observarla desde el punto de vista del efecto que produce; es decir, se intentan describir los procesos de recepción que surgen de la relación entre obra y lector. Como método de estudio literario, lo que hace la «Teoría o Estética de la Recepción» es fundamentar una nueva estética según la cual ambas caras del proceso de lectura, texto y lector, son inseparables.

${ }^{1}$ En el presente artículo se tienen en cuenta exclusivamente los dos primeros trabajos que iniciaron una nueva línea de investigación literaria: la «Estética de la Recepción». Esta teoría -todavía en estado provisional- ha experimentado un extraordinario desarrollo en sus veinte años de existencia; sin embargo, tanto la importancia y el interés de los textos programáticos de Jauss e Iser -pilares básicos de la corriente y, sin duda, todavía vigentes en buena medida-, como la imposibilidad de profundizar más en el corto espacio de este breve artículo, nos obliga a ceñirnos a la obra fundacional de los mencionados autores. Para un conocimiento totalmente actualizado sobre esta corriente de investigación véase: L. Acosta, El lector y la obra. Teoría de la recepción literaria. Gredos. Madrid, 1989. 
En este tema, Alemania presenta -a finales de los años 60- dos variantes, fundamentalmente ${ }^{2}$ : la encabezada por Jauss -que estudia el problema como diálogo entre obra y público- ha sido objeto de fuertes polémicas, mientras que los postulados de la segunda corriente, la protagonizada por Iser -que prefiere hablar de relaciones entre texto y lector- se han recogido en los planes de enseñanza y han alcanzado una efectividad práctica no lograda por Jauss, pese a haber tenido éste en los ámbitos intelectuales una mayor proyección.

Pasando por alto aquellos aspectos de la actual «Teoría de la Recepción» alemana cuya aplicabilidad a los cuentos de Grimm no presenta problemas, veamos -simplificando mucho las cosas- algunos de los planteamientos que, en principio, parecen vulnerables cuando se intentan aplicar al cuento que Propp denomina maravilloso y entre los que cabe clasificar a los de los Hermanos Grimm.

Hans Robert Jauss introdujo planteamientos innovadores, en lo que a recepción literaria se refiere, con su lección inaugural en la Universidad de Constanza, en 1967, bajo el título «Literaturgeschichte als Provokation der Literaturwissenschafi» (La Historia literaria como desafío a la Ciencia literaria). Su idea última gira en torno a la propuesta de una nueva descripción de la historia de la literatura a través de la visión que proporciona el lector, porque para Jauss la historicidad de la obra literaria depende absolutamente de su recepción, y, por tanto, la historicidad de la literatura está en íntima dependencia del lector; por su parte, la obra literaria predispone a su público a un preciso tipo de recepción mediante signos, características y otros elementos implícitos.

Jauss sostiene que en la relación entre obra y público se produce un diálogo continuado porque la lectura no es una mera reacción, sino una actividad consciente cuyo resultado es la formación del contenido histórico de la obra a través del lector. En este diálogo, que constituye el objeto de la «Estética de la Recepción», Jauss ve el dato primario para una historia de la literatura. Pero no es el propio proceso de lectura el que le preocupa, sino la determinación o precisión estética de la recepción. Partiendo de este primer dato, que es la relación entre obra y lector, observa, para establecer la historicidad de la literatura, dos aspectos que se derivan del acto de leer: la confrontación, a través de la propia posición de experiencia del lector, de sus ideas precedentes con la obra literaria, que sería el puro acto de lectura; y la comprensión, consistente en comparar la opinión aprehendida con el juicio de lectores anteriores.

¿Hasta qué punto -habría que preguntarse- es válido esto en el caso del cuento de Grimm? Si se tiene en cuenta que la inmensa mayoría de lectores de Grimm se compone de un público infantil ¿podemos hablar de confrontación de las ideas precedentes a través de la propia posición de experiencia del niño con el cuento? Naturalmente, Jauss se refiere muy probablemente al lector adulto y a la obra literaria escrita por un autor determinado. Y lo que observa es que se produce un choque de dos realidades: la del lector, su experiencia, sus ideas, y la que ese mismo lector detecta

${ }^{2}$ H.R. Jauss, Literaturgeschichte als Provokation der Literaturwissenschaft. Universitätverlag. Constanz 1967. W. Iser, Die Appellstruktur der Texte. Universitătverlag. Constanz 1970. 
en el acto de leer; pero, en el caso del niño, este problema tiene características específicas porque la frontera entre su realidad y la que le ofrece el cuento no está nada clara.

Por supuesto, se puede aceptar la tesis de un investigador como Bernd Wollenweber $^{3}$ y afirmar el carácter absolutamente realista del cuento al que nos estamos refiriendo. En tal sentido, la brutalidad del conflicto que plantean algunos cuentos de Grimm hace pensar en una verdadera historia criminal, como por ejemplo en «Hänsel und Gretel». El halo maravilloso que, sin embargo, envuelve a la narración o a los personajes, que, como bien dice Max Lüthi ${ }^{4}$, más que seres humanos, o tipos, son meras figuras, carentes frecuentemente de auténtica identidad personal, dota a la acción de extrema fantasía. Fantasía que el niño no percibe como exótica y ajena a su realidad cotidiana impregnada, a su vez, de buenas dosis de elementos fantásticos. Lo maravilloso se asume, por tanto, como real, y esto es consecuencia de la edad del lector, no del momento histórico en que vive o del entorno social que le circunda.

De poco serviría entonces, tratándose del cuento popular, confrontar -como propone Jauss- las ideas precedentes del lector con la obra literaria para establecer la historicidad de la literatura, porque entre la experiencia personal del niño y lo que el cuento le ofrece no hay una separación nítida.

Por lo que se refiere al otro aspecto, el que hacía referencia a la comparación de la opinión aprehendida con el juicio de lectores anteriores, parece sensato afirmar que el niño de cualquier época y país aprehende «Hänsel und Gretel» de similar manera; no hay lugar, pues, para establecer comparación alguna. El lector infantil, por su limitada capacidad intelectual, captará el contenido narrativo de forma parecida en todo tiempo y lugar.

Segundo tema importante en la concepción de Jauss es lo que denomina «horizonte de expectativa», es decir, aquello que el público espera de una obra de arte. El «horizonte de expectativa» de una obra hace posible determinar su carácter artístico por la forma en que es recibida y por el grado de efectividad producido en un público. Mediante la reconstrucción de este horizonte, se puede captar cómo fue entendida la obra por un lector de otra época; de esa manera, se muestra con claridad la diferencia de comprensión y captación pretérita y actual de una obra. Pues bien, Jauss cree que si una obra rompe el «horizonte de expectativa» exigiendo entonces un cambio de horizonte -como sucedió, por ejemplo, con la aparición en 1857 de Madame Bovary, de Flaubert- esa obra gana valor estético. $\mathrm{Si}$, por el contrario, cumple con las expectativas -sería el caso de Fanny, de Feydeau, publicada al mismo tiempo- estamos ante una obra de inferior valor.

Parece evidente que si esto es así, el cuento maravilloso, y por tanto el cuento de Grimm, es un arte de inferior valor, porque no cabe hablar de ruptura de horizonte de expectativa en este tipo de literatura especialmente ahistórica. ¿Cómo podríamos

${ }^{3}$ B. Wollenweber, «Thesen zum Märchen». En : S. Schödel (Hrsg.), Märchenanalysen. Reclam. Stuttgart 1983, pp. 62-69.

${ }^{4}$ M. Lüthi, «Wesenszüge des europäischen Volksmärchens». En: S. Schðdel(Hrsg.), Märchenanalysen. Reclam. Stuttgart 1983, pp. 47-55. 
hablar de lo que el público de la época de publicación de los cuentos de Grimm esperaba de éstos, si se trataba de una recopilación de algo ya conocido por tradición oral? La expectativa se produce ante lo desconocido, y tal requisito no se cumple en este tipo de literatura.

Según Jauss, hay que prestar atención, en primer lugar, al efecto que la obra en cuestión surtió en su momento, pero ¿cuál es ese momento en literatura popular de tradición oral? Luego -dice Jauss- hay que observar la obra en una nueva forma desde la perspectiva del presente, nueva forma proporcionada por el lector actual; y también aquí cabe la pregunta ¿es que el niño actual recibe el cuento de Grimm de manera nueva y distinta? Por consiguiente, el argumento de que para dar sentido a la historia de la literatura es necesario reunir los testimonios del efecto producido hasta el momento y observarlos desde la perspectiva que confiere la posición del presente no parece válido en el caso de los cuentos, porque si nos referimos a éstos, la historia de la recepción ya no es el sucesivo desarrollo de una obra cuyos diferentes grados de sentido se van actualizando hasta formar un juicio, como define Jauss; la resultante que se produce entre tradición y horizonte en cualquier otro tipo de literatura carecería de sentido en el supuesto del cuento maravilloso.

Un último elemento citado por Jauss como indispensable para completar la tarea de la historia de la literatura es el poder relacionar el fenómeno literario con la historia general. Y aquí la inoperancia de su teoría aplicada al cuento se hace, si cabe, más evidente porque el cuento es intemporal y ahistórico por excelencia, contrariamente a la saga, por ejemplo, más ligada a un lugar y a un momento histórico. Naturalmente, toda obra de ate es en algún sentido ahistórica, en otro caso no podría ser objeto de recepción ulterior; los valores estéticos de un texto literario, cuanto menos ligados estén a momentos históricos concretos, más posibilidades tienen de poder ser actualizados posteriormente. Lo que sucede con el cuento de Grimm es que son muy escasos los indicios que permiten establecer conexiones con un tiempo histórico determinado; y por eso, el elemento invocado por Jauss de conjunción histórica y literaria no parece tener aplicabilidad en este tipo de literatura.

De lo dicho hasta aquí, hay que pensar que si la «Teoría de la Recepción» sirve, en opinión de Jauss, no sólo para captar el sentido y la forma de la obra literaria, sino también su conexión dentro de la historia de la literatura, difícilmente ambos objetivos serían alcanzables tratándose de literatura cuentista.

La segunda variante de la «Estética de la Recepción» alemana en su época fundacional es la que protagoniza Wolfgang Iser y que plasma en su obra «Die Appellstruktur der Texte» (La estructura apelativa del texto). La importancia de la obra literaria -se dice ahí- surge con el proceso de lectura, que es el producto de una interacción entre texto y lector. Iser reduce los textos literarios a su estructura, a su forma indeterminada, que lo es por estar vacía de contenido significativo. El significado ha de esclarecerse apelando al lector. Se reduce, pues, la obra literaria a lo dado; es decir, a la conexión que establece un conjunto de palabras y frases. En este sentido, la obra es simplemente forma, texto preformado ante el cual reacciona el lector que, con su peculiar manera de recibir el mensaje textual, conforma y completa la obra literaria en su conjunto; la esteticidad del texto está, por tanto, en íntima 
conexión con la libertad del lector, dice Iser; el lector no precisa de conocimientos especiales previos, simplemente necesita haber adquirido la capacidad que le permita enfrentarse al texto como esctructura. Naturalmente, como el propio lector es quien genera el significado, éste adopta forma individual y carece por lo tanto de objetividad.

A partir de aquí aparecen ya puntos vulnerables si se piensa en cuentos como los de Grimm, ya que esa falta de objetividad obliga -en opinión de Iser- a someter los significados a un continuo proceso de actualización, actualización que realiza el propio lector. Nosotros actualizamos un texto -dice Iser- cada vez que lo leemos porque cada texto encierra en sí diferentes posibilidades de actualización, puesto que ha sido entendido con matices distintos en diferentes momentos históricos y por lectores muy diversos. Sin embargo, según hemos dicho, no parece que «Hänsel und Gretel», por ejemplo, se haya entendido de forma diferente por lectores de distintas épocas. Otra cosa es que el análisis literario pueda mostrar multitud de interpretaciones observables en un cuento de Grimm como en cualquier otro texto; lo cual en absoluto significa que el cuento en sí experimente constantes actualizaciones mediante las lecturas que de él hace su público infantil.

Por otra parte, de acuerdo con Iser, los contenidos semánticos están en función del entorno, pero como el texto no tiene relación directa con el exterior -continúa Iserpuesto que es ficción lo que contiene, tal relación surge mediante el contacto con el lector, cuya mediación produce ya un efecto de clarificación de la relativa indeterminación del texto literario. Así pues, el mundo del texto se enfrenta, por un lado, al mundo de las experiencias del lector; y, por otro, al mundo exterior, al mundo de los objetos reales pero a través del propio lector. Dejando aparte la cuestión en sí (discutible, sin duda, porque Iser olvida por completo al autor, y, por lo tanto, la relación estrecha entre texto y entorno a través de él), y ciñéndonos al cuento de Grimm, si el contenido semántico de los cuentos está en función del entorno y es a través del lector como se generan los significados, habría que recurrir a la Sicología Social para desvelar el misterio de cómo es posible que el niño interiorice realidades tan diferentes -la del cuento y la que observa desde que nace-, y cómo es posible que a partir de su entorno genere significados maravillosos en un texto vacío de contenidos. Tal vez, sin embargo, una explicación sea que la realidad del niño -como decíamos al comentar a Jauss- es una mezcla de objetos reales y elementos maravillosos.

En fin, a la vista de lo expuesto, pueden apuntarse las siguientes conclusiones:

1) La preponderancia que la «Estética de la Recepción» concede al lector para determinar el valor, la historia o el significado literario cojea cuando se trata de literatura cuentista popular, porque en ella el lector es más destinatario que creador.

2) La ausencia de autor individual en el cuento de Grimm dificulta la relación autor-lector. Al no existir un autor diferenciado e individualizado, el rasgo autobiográfico, presente en cualquier obra literaria, desaparece. El caso de estos cuentos es, pues, para la «Teoría de la Recepción» similar, en cierto modo, al de la lírica primitiva: se trata de actividades colectivas -en lo que se refiere a su composición y, quizá en menor medida, también a su recepción- en las que ambas partes de la relación (autorlector) no presentan signos diferenciadores específicos como consecuencia de sus 
propias y genuinas peculiaridades: obras fruto de la creación anónima colectiva y destinadas a transmitirse oralmente.

3) En el cuento los datos están permanentemente actualizados. Por tratarse de un producto ahistórico, así como por su enorme grado de ficción, no tiene sentido el plantearse problemas de inmersión y captación del entorno, es decir, tratar de esclarecer la época en que surgió o aquella en la que transcurre la narración.

4) El significado -que en otro tipo de literatura puede generarlo en gran medida el lector- es en el cuento consustancial a la obra misma por las peculiares características del público al que va destinado: el niño. Por eso, la recepción del texto entendida como generación actual de significados estéticos apenas tiene sentido cuando nos referimos al cuento popular.

5) En el cuento se puede hablar de concreción del valor literario e incluso de interpretación, pero no de recepción en el sentido de una recepción distinta.

6) Más que de recepción, habría que hablar -cuando se trata de literatura cuentista de carácter popular- por un lado de actualidad, que muy probablemente es la misma en tiempos de Jacob y Wilhelm, en el siglo XIV o en 1989, porque los contenidos son perfectamente asumibles por el lector de cualquier época. Y, por otro lado, de vigencia, aspecto más discutible en un mundo en el que las historias de galaxias y las fantasías del campo de la técnica parecen haber arrinconado determinados valores estéticos tradicionales.

7) Si la ciencia de la literatura es una ciencia de comunicación y relaciones que exige la conexión autor-texto-lector, así como los procesos que entre ellos tienen lugar, es obvio que la obra literaria no es ni estática ni objetiva; su comprensión ha de realizarse desde la perspectiva subjetiva como un factor variable cuyo valor depende de la interacción con otros factores. Pero esto, que sin duda es válido para la literatura de creación en general, no lo es tanto en el caso de los cuentos de Grimm y del cuento popular de cualquier época: su grado de dinamismo y subjetividad se reduce en gran manera precisamente por su rasgo de ahistoricidad, intemporalidad y elevado grado de ficción. Sincronía y diacronía -vías propuestas por Jauss para el estudio de la recepción- dejan de ser relevantes en este tipo de cuentos, de forma que lo que interesa es un análisis textual inmanente que permita su interpretación.

En resumen: la actual «Estética de la Recepción» no se agota con las ideas de Jauss e Iser tenidas en cuenta en este breve trabajo. Ambos autores, junto con otros investigadores, continúan ocupándose del tema tanto en la República Federal de Alemania como en la República Democrática Alemana. Sin embargo, como método de estudio literario se encuentra todavía en fase inicial. Es de esperar, por tanto, que en el futuro se aborde el estudio de la literatura popular bajo las orintaciones y enfoques de la «Teoría de la Recepción». En todo caso, y ya que el cuento popular presenta elementos que lo alejan de otros tipos de literatura, lo que sí se puede afirmar -ateniéndonos al estado inicial de las investigaciones- es que el cuento de Grimm difícilmente encaja en lo que propugna la «Estética de la Recepción». Si esto llegara a confirmarse, parecería entonces más lógico integrar el cuento popular en un campo de estudio que abarque otras manifestaciones o expresiones artísticas de carácter popular, como sucede actualmente en Alemania con el estudio de la «Volkskunde». 